\title{
A relação entre os mecanismos de defesa e a qualidade da aliança terapêutica em psicoterapia de orientação analítica
}

\author{
The relationship between defense mechanisms and the quality of therapeutic \\ alliance in analytic psychotherapy
}

\begin{abstract}
Fernando Grilo Gomes ${ }^{1}$, Lucia Helena Ceitlin², Simone Hauck ${ }^{3}$, Luciana Terra ${ }^{4}$
${ }^{1}$ Psiquiatra psicanalista. Professor adjunto, Departamento de Psiquiatria e Medicina Legal, Faculdade de Medicina (FAMED), Universidade Federal do Rio Grande do Sul (UFRGS), Porto Alegre, RS. 2 Psiquiatra. Professora adjunta, Departamento de Psiquiatria e Medicina Legal, FAMED, UFRGS, Serviço de Psiquiatria, Hospital de Clínicas de Porto Alegre (HCPA), Porto Alegre, RS. Professora, Pós-Graduação em Clínica Médica: Psiquiatria, UFRGS. ${ }^{3}$ Psiquiatra. Mestre em Psiquiatria, UFRGS. Médica contratada, Serviço de Psiquiatria, HCPA. ${ }^{4}$ Psicóloga. Mestranda em Psiquiatria, UFRGS Este estudo foi realizado no Serviço de Psiquiatria do Hospital de Clínicas de Porto Alegre (HCPA), Porto Alegre, RS.
\end{abstract}

\section{Resumo}

Introdução: $\mathrm{O}$ estabelecimento de uma aliança terapêutica de boa qualidade em uma psicoterapia psicanalítica é fundamental para o processo terapêutico. Este estudo avaliou a influência do nível de funcionamento defensivo do paciente na qualidade da aliança terapêutica estabelecida durante a psicoterapia.

Método: Para avaliação da qualidade da aliança estabelecida, pacientes em psicoterapia psicanalítica e seus respectivos terapeutas responderam ao Helping Alliance Questionnaire (versão paciente e versão terapeuta, respectivamente). O nível defensivo foi inferido através da Escala de Funcionamento Defensivo proposta no Manual de Diagnóstico e Estatística das Perturbações Mentais, quarta edição, texto revisado.

Resultados: Não houve associação entre o estabelecimento de uma aliança terapêutica de boa qualidade e o nível defensivo do paciente. No entanto, houve diferença significativa quando a versão do terapeuta foi comparada com a respondida pelo paciente: os pacientes estabeleceram uma aliança terapêutica de melhor qualidade em relação a seus terapeutas do que o inverso.

Conclusão: $O$ fato de a aliança terapêutica de boa qualidade ter se estabelecido independentemente do nível defensivo do paciente sugere que o treinamento e as características pessoais do terapeuta podem levar a uma capacidade de conectar-se com o paciente, apesar do grau de comprometimento do seu funcionamento psíquico.

Descritores: Psicoterapia, psicanálise, mecanismos de defesa, aliança terapêutica.

\begin{abstract}
Introduction: The quality of a therapeutic alliance is essential in psychoanalytic psychotherapy and influences the therapeutic process. This study evaluated the relationship between the level of defense mechanisms and the quality of therapeutic alliance established during psychotherapy.

Method: Patients in psychotherapy and their respective therapists completed the Helping Alliance Questionnaire (patient version and therapist version, respectively). The level of defenses was inferred by the Defensive Functioning Scale proposed in the Diagnostic and Statistical Manual of Mental Disorders, fourth edition, text revision.

Results: There was no association between the quality of therapeutic alliance and the patient's level of defense mechanisms in this sample. On the other hand, there was a difference when the therapist version was compared to the patient version: patients established a stronger therapeutic alliance in relation to their therapists.

Conclusion: The lack of influence of defense level in the quality of therapeutic alliance suggests that the therapist's training and personal characteristics may lead to the ability of connecting with the patient, despite impairment in their psychic functioning. Keywords: Psychotherapy, psychoanalysis, defense mechanisms, therapeutic alliance.
\end{abstract}




\section{Introdução}

A psicoterapia psicanalítica (PSCPA) é uma modalidade de tratamento psicológico que se baseia na teoria e na técnica da psicanálise, utilizando-se do conceito de inconsciente postulado por Freud ${ }^{1}$, da associação livre e da compreensão dos sonhos, do humor e dos atos falhos para entender e dar um significado aos conflitos inconscientes. No entanto, embora considere o padrão transferencial/contratransferencial no entendimento do paciente e na elaboração das interpretações, diferentemente da psicanálise que se caracteriza pelo uso do divã, por uma maior freqüência de sessões (três a cinco sessões por semana) e pelo uso freqüente da interpretação transferencial, a PSCPA utilizase predominantemente de interpretações extratransferenciais e de uma freqüência menor de sessões (uma a duas por semana), além de não fazer uso do divã.

Ao longo dos anos, diversos estudos vêm tentando definir os fatores que estariam associados a um melhor desfecho em PSCPA, com o intuito de melhorar a indicação desse tipo de tratamento e aprimorar a técnica ${ }^{2-}$ ${ }^{6}$. Dentre os aspectos que vêm sendo ressaltados como bons indicadores de prognóstico, a aliança terapêutica (AT), primeiramente delineada por Freud ${ }^{7}$ em 1912 como transferência positiva, parece ter papel central ${ }^{2,8}$.

Define-se AT como uma relação positiva e estável entre terapeuta e paciente, que permite levar a cabo uma psicoterapia de orientação analítica. Segundo Melanie Klein ${ }^{9}$, essa aliança origina-se a partir das relações precoces de objeto, sendo necessária, para sua construção, pelo menos uma experiência na qual foi possível interagir com outra pessoa de modo saudável, como, por exemplo, uma relação afetiva e "continente" do bebê com seu cuidador (comumente citada como relação mãe-bebê). Marcolino ${ }^{10}$ constatou que uma melhor qualidade da AT esteve associada a melhores resultados e a um grau maior de diminuição da sintomatologia em pacientes em um programa de psicoterapia breve. Kaplan ${ }^{11}$ sugere que nenhuma análise pode avançar sem o estabelecimento de uma boa AT, sendo necessária, para o seu estabelecimento, a existência, dentro da mente do paciente, de uma parte capaz de se manter livre de conflitos e racional, que se distancie a fim de reconhecer a natureza irracional de outra parte de sua mente que inconscientemente repete os conflitos psíquicos.

Um dos aspectos freqüentemente mencionado como determinante da qualidade da AT é a personalidade do paciente, que, dentre outros aspectos, manifesta-se através do padrão defensivo do mesmo. Fadiman ${ }^{12}$ argumenta que os mecanismos de defesa são um conjunto de operações que permitem reduzir ou suprimir estímulos que possam causar desprazer, tentando, assim, manter o equilíbrio do aparelho psíquico. O uso de mecanismos de defesa está presente em todas as pessoas e é vital para o funcionamento psíquico. O que define uma melhor ou pior capacidade adaptativa é a natureza, a intensidade e a freqüência do uso de mecanismos de defesa mais, ou menos, maduros.

George Vaillant ${ }^{13}$, em seu livro Adaptation to life, reporta-se aos trabalhos de Freud e Anna Freud para, no capítulo 5, "Mecanismos adaptativos do ego, uma hierarquia", classificar 18 mecanismos de defesa em quatro níveis: mecanismos psicóticos (comuns nas psicoses, sonhos e nas crianças pequenas), mecanismos imaturos (comuns nas depressões severas, transtornos de personalidade e adolescência), mecanismos neuróticos (comuns em todas as pessoas) e mecanismos maduros (comuns em adultos sadios), de acordo com a evolução do processo adaptativo, sua maturidade e importância patológica. Sugere, ainda, que o amadurecimento da vida humana é acompanhado pela evolução destes processos adaptativos e que a hierarquia descrita não reflete somente um continuum da criança ao adulto, mas também da doença até a saúde. Nesse mesmo sentido, Kipper ${ }^{14}$ evidenciou, em pesquisa recente, que pacientes com pânico faziam uso de mecanismos de defesa mais desadaptativos comparados com controles normais, assim como diferenças após o tratamento psicofarmacológico.

Embora exista, a priori, uma hipótese teórica de que o uso de mecanismos de defesa mais maduros facilitaria o estabelecimento da AT, alguns estudos, como um estudo multicêntrico realizado por Hersoug ${ }^{15}$ que investigou a associação entre os mecanismos de defesa utilizados pelo paciente e a qualidade da AT, demonstraram que o padrão defensivo não influenciou nem a qualidade da AT nem a melhora em psicoterapia breve dinâmica, concluindo que os sintomas cedem no início da terapia e que as defesas podem mudar ao longo do tratamento.

O presente estudo busca investigar, em uma amostra de pacientes atendidos no Programa de Psicoterapia Psicanalítica do Hospital de Clínicas de Porto Alegre (HCPA), a relação entre a capacidade do paciente em estabelecer uma AT (conforme a percepção do próprio paciente e do seu terapeuta) e o nível dos mecanismos de defesa inconscientes utilizados pelo paciente naquele momento do tratamento.

\section{Método}

O delineamento do estudo foi transversal, sendo a amostra selecionada por conveniência: foram convidados a participar os pacientes que estavam realizando PSCPA no Ambulatório de Psicoterapia do HCPA no período de realização do estudo e seus terapeutas - residentes do Serviço de Psiquiatria do HCPA e alunos do segundo e terceiro ano do curso de especialização em Psiquiatria do Departamento de Psiquiatria e Medicina Legal da Faculdade de Medicina (FAMED) da Universidade Federal do Rio Grande do Sul (UFRGS). 
Os pacientes foram encaminhados para o Programa de Psicoterapia por outros ambulatórios do HCPA, inclusive o ambulatório de triagem (que recebe os pacientes encaminhados da rede municipal de saúde de atendimento primário), quando havia uma possível indicação de PSCPA. A indicação foi confirmada pelo terapeuta com auxílio de seu supervisor, levando em consideração os critérios descritos por Cordioli ${ }^{16}$, como existência de um foco de trabalho, capacidade de pensar psicologicamente, motivação para realização de PSCPA, nível de adaptação prévio, entre outros. Os pacientes foram alocados para os terapeutas de forma aleatória de acordo com a ordem de chegada no ambulatório e disponibilidade de horários.

Os terapeutas realizaram atividades de supervisão permanente ao longo do estudo, orientados por supervisores com, no mínimo, 15 anos de experiência em PSCPA: 1 hora de supervisão individual semanal, além de uma supervisão semanal em grupo de 80 minutos, na qual um paciente era entrevistado por um dos supervisores, sendo o caso discutido, logo a seguir, quanto ao diagnóstico e planejamento terapêutico. Todos os terapeutas foram treinados para a aplicação das escalas, sendo que a AT e os sintomas do paciente foram inferidos por meio de escalas autopreenchidas, e os mecanismos de defesa, por questionário estruturado proposto no Manual de Diagnóstico e Estatística das Perturbações Mentais, quarta edição, texto revisado $(\mathrm{DSM}-\mathrm{IV}-\mathrm{TR})^{17}$.

Exceto a indicação de PSCPA, não houve critérios de exclusão. Todos os pacientes que participaram do estudo e seus respectivos terapeutas assinaram consentimento livre e esclarecido aprovado pelo Comitê de Ética do HCPA.

Para avaliar os mecanismos de defesa, utilizou-se a Escala de Funcionamento Defensivo do DSM IV-TR ${ }^{17}$, em que o terapeuta deve escolher, pontuando de forma hierárquica, os sete mecanismos de defesa usados com maior freqüência dentre os 25 listados. O funcionamento defensivo do paciente é classificado, posteriormente, em um dos seguintes níveis: alto nível adaptativo, nível de inibições mentais (formação de compromisso), nível de leve distorção da imagem, nível da negação, nível de importante distorção da imagem, nível da ação e nível da desregulação defensiva. Um glossário padronizado é utilizado para homogeneizar a conceitualização de cada um dos mecanismos de defesa, bem como dos diferentes níveis defensivos ${ }^{16}$. Estes sete níveis foram posteriormente agrupados em níveis de defesas maduras (nível 1), intermediárias (nível 2) e imaturas ou primitivas (nível 3).

O diagnóstico utilizado foi o registrado no prontuário dos pacientes, utilizando os critérios do DSM-IV-TR ${ }^{17}$. O diagnóstico foi realizado pelos terapeutas, com a supervisão dos respectivos preceptores, a partir das entrevistas clínicas de avaliação diagnóstica e indicação terapêutica, utilizando-se o último registro realizado no prontuário hospitalar de cada paciente.

A AT foi avaliada pelo instrumento Helping Alliance Questionnaire (HAq II) ${ }^{18}$, versão do paciente e do terapeuta, elaborado por Luborsky et al. em 1996, que consiste em um questionário auto-respondido que contém 19 itens que avaliam, em uma escala tipo Likert (1 a 6), dimensões essenciais do relacionamento paciente-terapeuta, tais como sentimentos de compreensão, confiança, interesse, objetivos comuns e desejo de progresso. Esta escala foi traduzida e retraduzida por professores de inglês/português com conhecimento na área da pesquisa, testada e adaptada conforme as normas do Grupo de Pós-Graduação e Pesquisa (GPPG) do HCPA, sendo aprovada e autorizada, em sua versão final adaptada para português brasileiro, pelo autor do instrumento original.

A análise estatística foi realizada através do SPSS 13.0 com o auxílio do GPPG do HCPA. Os grupos foram comparados através do teste $t$ de Student e o teste exato de Fisher, enquanto as correlações foram investigadas através dos testes de Pearson e Spearman.

\section{Resultados}

A amostra foi composta por 37 duplas (pacientes/ terapeutas), sendo que, em três das 40 duplas que realizaram PSCPA no Ambulatório do Serviço de Psiquiatria de Adultos do HCPA no período da pesquisa, os pacientes não concordaram em participar do estudo.

A composição da amostra de pacientes ficou assim caracterizada: a maioria eram mulheres (27), a média de idade foi de 38 anos (desvio padrão - DP = 13), e todos eram alfabetizados. Em relação à situação conjugal, 45,9\% eram casados, 35,1\%, solteiros, e $18,9 \%$, separados.

A composição da amostra de terapeutas foi assim constituída: os terapeutas que participaram do estudo foram em número de 12 residentes (residência em Psiquiatria do HCPA) ou cursistas (curso de especialização em Psiquiatria do Departamento de Psiquiatria e Medicina Legal, FAMED, UFRGS), estando seis no segundo ano de formação e seis no terceiro ano, resultando em um mínimo de 1 ano e meio de formação psiquiátrica. Sete terapeutas eram do sexo masculino, e cinco, do sexo feminino. Como os terapeutas atenderam mais de um paciente durante o período, deduz-se que cada terapeuta participou de mais de uma dupla.

Considerando o nível defensivo dos pacientes, 11 deles utilizavam predominantemente defesas do nível 1 (grupo de defesas maduras), 15 pacientes, do nível 2 
(grupo de defesas intermediárias), e 11 pacientes, do nível 3 (grupo das defesas primitivas).

O diagnóstico, segundo o DSM-IV-TR, foi realizado a partir de entrevista clínica e discutido em supervisão, estando descrita a freqüência de diagnósticos nos eixos I (Tabela 1) e II (Tabela 2).

Quanto à freqüência de sessões, a maioria dos pacientes $(59,5 \%)$ realizava duas sessões semanais, ao passo que $40,5 \%$ realizavam uma sessão semanal. $\mathrm{Na}$ análise da percepção pelos pacientes de aliança forte ou fraca conforme número de sessões semanais (uma ou duas), não houve diferença significativa $(\mathrm{p}=0,266)$. $\mathrm{O}$ mesmo foi demonstrado para os escores totais na escala versão terapeuta $(\mathrm{p}=0,897)$. Dessa forma, a percepção da qualidade da AT forte ou fraca pelos pacientes e terapeutas foi independente do número de sessões semanais.

Em relação ao tempo de terapia, 25 pacientes $(67,5 \%)$ estavam em terapia há mais de 6 meses. Estar

Tabela 1 - Diagnósticos em eixo I

\begin{tabular}{lcc}
\hline Diagnósticos & Freqüência & Porcentagem \\
\hline Sem diagnóstico & 9 & 24,3 \\
Abuso/abstinência de álcool & 1 & 2,7 \\
Transtorno de ansiedade generalizada/depressão & 1 & 2,7 \\
Transtorno bipolar/anorexia nervosa & 1 & 2,7 \\
Transtorno depressivo maior & 10 & 27,0 \\
Transtorno depressivo moderado & 2 & 5.4 \\
Transtorno distímico & 1 & 2,7 \\
Transtorno afetivo bipolar em remissão & 3 & 8,1 \\
Síndrome de ansiedade & 1 & 2,7 \\
Transtorno de ansiedade generalizada & 1 & 2,7 \\
Transtorno do pânico com agorafobia & 1 & 2,7 \\
Transtorno afetivo bipolar 1 & 1 & 2,7 \\
Transtorno afetivo bipolar2 & 1 & 2,7 \\
Transtorno afetivo bipolar 3 & 1 & 2,7 \\
Transtorno obsessivo-compulsivo & 2 & 5,4 \\
Transtorno do pânico & 1 & 2,7 \\
Total & 37 & 100,0 \\
\hline
\end{tabular}

Tabela 2 - Diagnósticos em eixo II

\begin{tabular}{lcc}
\hline Diagnósticos & Freqüência & Porcentagem \\
\hline Sem diagnóstico & 15 & 40,5 \\
Transtorno de personalidade depressiva & 2 & 5.4 \\
Transtorno de personalidade evitativa/fóbica & 1 & 2,7 \\
Transtorno de personalidade histriônica & 5 & 13,5 \\
Luto patológico & 1 & 2,7 \\
Transtorno de personalidade obsessiva & 2 & 5,4 \\
Transtorno de personalidade dependente & 1 & 2,7 \\
Transtorno de personalidade esquizóide & 1 & 2,7 \\
Traços histriônicos & 2 & 5,4 \\
Traços narcisistas & 3 & 8,1 \\
Traços histriônicos/masoquistas & 1 & 2,7 \\
Traços histriônicos/obsessivos & 1 & 2,7 \\
Traços obsessivos & 2 & 5,4 \\
Total & 37 & 100,0 \\
\hline
\end{tabular}


em psicoterapia há mais de 6 meses esteve associado a uma percepção maior de aliança forte $(\mathrm{p}=0,016)$, indicando que, quanto maior o tempo de terapia, melhor foi considerada a AT pelos pacientes. $\mathrm{Na}$ análise da percepção dos terapeutas, houve uma tendência de um resultado semelhante $(\mathrm{p}=0,053)$.

Houve uma diferença estatisticamente significativa $(\mathrm{p}=0,001)$ em relação à qualidade da AT desenvolvida por pacientes de sexos diferentes em relação a seus terapeutas, sendo que os pacientes homens perceberam o estabelecimento de uma aliança mais forte do que a referida pelas pacientes mulheres. No entanto, quando considerado o escore total da AT percebida pelo terapeuta em relação ao sexo do paciente, não houve diferença significativa $(\mathrm{p}=0,50)$.

De acordo com resultados encontrados por outros pesquisadores ${ }^{19}$, não houve correlação entre o estilo defensivo dos pacientes e a qualidade da AT percebida pelos mesmos $(p=0,797)$ ou pelos terapeutas $(p=$ 0,925). A ausência de significância se manteve mesmo quando as dimensões da escala HAq II foram consideradas separadamente.

A percepção da AT pelo paciente foi significativamente mais "forte" (escores maiores) do que pelo terapeuta, sendo esse achado significativo em 11 das 19 questões da HAq II, quando analisadas em separado.

\section{Discussão}

A ausência de associação entre o padrão defensivo do paciente e a formação da AT poderia sugerir que essa variável está de fato mais relacionada a fatores ditos "inespecíficos", reforçando a importância dos fatores relacionados ao terapeuta em relação às variáveis determinantes de prognóstico em PSCPA $^{19-23}$.

Esses achados também apontam para a consideração de outras hipóteses: será possível que o treinamento recebido pelos terapeutas possa ter levado ao desenvolvimento de uma capacidade qualificada de interação com seus pacientes independentemente do diagnóstico, da sintomatologia e dos níveis dos mecanismos de defesa?

Despland ${ }^{24}$, em sua pesquisa a respeito dos mecanismos de defesa e das intervenções do terapeuta no desenvolvimento de uma AT precoce, refere que a aliança desenvolve-se rapidamente, em torno da terceira sessão, independentemente das intervenções ou das defesas do paciente. Entretanto, o ajustamento das intervenções do terapeuta ao nível dos mecanismos de defesa do paciente poderia melhorar a qualidade da AT. O ajustamento das intervenções terapêuticas parece ser, de fato, um fator promotor do desenvolvimento da AT.
Por outro lado, Ackerman ${ }^{25}$ demonstrou, em sua pesquisa sobre a influência das características do terapeuta e de sua técnica no desenvolvimento da AT, que algumas características do terapeuta, como flexibilidade, respeito, honestidade, confiabilidade, confidência, calor humano, interesse e tolerância, contribuíram positivamente para a formação da AT. Além disso, o uso de técnicas de exploração, reflexão, valorização de resultados, interpretações acuradas, facilitação da expressão dos afetos e valorização da experiência do paciente também contribuiu positivamente na aliança.

Outro ponto a ser considerado é o fato de os pacientes serem examinados por terapeutas com características específicas (com idade, sexo, experiência e características pessoais diversas), o que poderia comprometer tanto a validade interna (pelas diferenças entre eles) como a validade externa, por se tratar de terapeutas jovens.

Poderia o fato de se tratar de terapeutas jovens, em início de carreira, propiciar um maior investimento na AT e uma maior disposição? Malann ${ }^{26}$, em sua pesquisa pioneira sobre psicoterapia breve dinâmica, evidenciou que o "entusiasmo" dos terapeutas jovens era o fato mais importante para a obtenção de resultados positivos.

Poderíamos considerar várias limitações no presente estudo. Embora todos os casos tenham sido avaliados de forma subjetiva conforme os critérios referidos por Cordioli ${ }^{16}$ e discutidos com os supervisores, não é possível afirmar com certeza absoluta se todos os pacientes teriam de fato indicação de PSCPA. Outro fator a ser apontado, mesmo considerando um criterioso processo de adaptação do HAq II para o português brasileiro, é a ausência de dados de validação em nosso meio que pudessem confirmar o comportamento do instrumento na nossa população (análise fatorial, análise confirmatória e testes de confiabilidade).

Como não houve uma medida de adesão baseada em sessões gravadas ou filmadas, outro fator a ser considerado é a especificidade da técnica utilizada pelos terapeutas: foram de fato utilizadas exclusivamente técnicas de PSCPA ou ocorreu também o uso de técnicas cognitivas ou de apoio com uma compreensão analítica, apesar da supervisão sistemática? Nesse caso, os resultados encontrados poderiam ter sofrido influência dessas variáveis. Também o número de duplas envolvidas nessa amostra pode ser considerado pequeno, mesmo considerando a magnitude dos resultados e concordância com outros estudos, o que poderia levar a uma limitação da validade externa por um aumento da chance de erro tipo beta.

A não-existência de um manual de PSCPA poderia resultar em um comprometimento da uniformidade do 
procedimento; no entanto, vale lembrar que Luborski ${ }^{27,28}$ ressaltou que, em PSCPA, o uso de um manual e de parâmetros muito rígidos poderia estreitar o planejamento da psicoterapia e que realmente deve-se considerar que um dos maiores agentes para a efetividade de uma psicoterapia é a personalidade do terapeuta.

É possível que o estabelecimento de um AT de melhor qualidade não dependa exclusivamente do nível dos mecanismos de defesa usados e sim do tipo de relação transferencial estabelecida, da necessidade do paciente e da disponibilidade do terapeuta, apontando a necessidade de estudos que considerem essas varáveis na investigação dos fatores promotores de uma AT de qualidade.

\section{Conclusão}

Os achados deste estudo demonstraram não haver uma correlação significativa entre os mecanismos de defesa utilizados pelos pacientes e a capacidade de estabelecer uma AT de boa qualidade, confirmando achados de outros estudos que sugerem serem os fatores ditos "inespecíficos" mais importantes, inclusive, que as características do paciente e a técnica utilizada ${ }^{19-23}$. Surge, então, a partir dessa hipótese, a necessidade (e o desafio) de elaborar instrumentos e metodologias de pesquisa que viabilizem a caracterização e a investigação do real impacto de tais fatores.

\section{Agradecimentos}

Ao Professor Doutor Lester Luborsky, pela gentileza de autorizar o uso do instrumento HAq II; aos residentes e cursistas em Psiquiatria que participaram deste trabalho; e aos Professores Doutor Cláudio Laks Eizirik e Doutora Psicóloga Maria Lúcia Tillet Nunes, por sua ajuda e colaboração com seus comentários valiosos.

\section{Referências}

1. Freud S. O inconsciente. Rio de Janeiro: Imago; 1915.

2. Barber JP, Connoly MB, Crits-Christoph P, Gladis L, Siqueland L. Alliance predicts patients' outcome beyond in-treatment change in symptoms. J Consult Clin Psychol. 2000;68:1027-32.

3. Ceitlin LHF, Wiethaeuper D, Goldfeld PRM. Pesquisa de resultados em psicoterapia de orientação analítica: efeitos das variáveis do terapeuta. Rev Bras Psicoter. 2003;5:81-95.

4. Luborsky L, Crits-Christoph P, Mintz J, Auerbach A. Who will benefit from psychotherapy? New York: Basic Books; 1988.
5. Nathan PE, Stuart SP, Dolan SL. Research on psychotherapy efficacy and effectiveness: between Scylla and Charybdis. Psychol Bull. 2000;126:964-81.

6. Valbak K. Suitability for psychoanalytic psychotherapy: a review. Acta Psychiatr Scand. 2004;109:164-78.

7. Freud S. A dinâmica da transferência (1912). In: Edição standart brasileira das obras psicológicas completas de Sigmund Freud. Rio de Janeiro: Imago; 1976.

8. Martin DJ, Garske JP, Davis MK. Relation of the therapeutic alliance with outcome and other variables: a meta-analytic review. J Consult Clin Psychol. 2000;68:438-50.

9. Klein M. Fundamentos psicológicos da análise infantil (1926). In: Psicanálise da criança. São Paulo: Mestre Jou; 1981.

10. Marcolino JAM, Iacoponi E. The early impact of therapeutic alliance in brief psychodynamic psychotherapy. Rev Bras Psiquiatr. 2003;25:78-86.

11. Kaplan HI. Psicoterapias. Porto Alegre: Artmed; 1997:773

12. Fadiman J, Frager R. Teorias da personalidade. São Paulo: Harbra; 1980.

13. Vaillant GE. Adaptation to life. Cambridge: Harvard University; 2001.

14. Kipper LC. Avaliação de mecanismos de defesa em pacientes com transtorno de pânico, sua relação com gravidade, resposta ao tratamento e alteração pós-tratamento [dissertação] Porto Alegre: UFRGS; 2003.

15. Hersoug AG, Sexton HC, Hoglend P. Contribution of defensive functioning to the quality of working alliance and psychotherapy outcome. Am J Psychoter. 2002;56:539-54.

16. Cordioli AV. Psicoterapias: abordagens atuais. Porto Alegre: Artmed; 1998.

17. American Psychiatric Associaton. In: DSM-IV-TR ${ }^{\mathrm{TM}}$ - Manual diagnóstico e estatístico de transtornos mentais. Porto Alegre: Artmed; 2002:754-9.

18. Luborsky L, Barber JP, Siqueland L, Johnson S, Najavits LM, Frank A, et al. The revised helping alliance questionnaire (Haq-II). J Psychoter Pract Res. 1996;5:260-71.

19. Berzins JI. Therapist-patient matching. In: Gurman AS, Razin AM, eds. Effective psychotherapy: a handbook of research. Elmsford: Pergamon; 1977. p. 222-51.

20. Beutler LE, Machado PPP, Neufeldt S. Therapist variables. In: Garfield SL, Bergin AE, eds. Handbook of psychotherapy and behavior change. New York: John Wiley \& Sons; 1994. p. 259-69.

21. Gelso CJ, Mills DH, Spiegel SB. Client and therapist factors influencing the outcomes of time-limited counseling one month and eighteen months after treatment. In: Gelso CJ, Johnson DH, eds. Explorations in time limited counseling and psychotherapy New York: Teachers College; 1983. p. 87-114.

22. Hayes JA. The inner world of the psychotherapist: a program of research on countertransference. Psychother Res. 2004;14:21-36.

23. Kelly TA, Strupp HH. Patient and therapist values in psychotherapy: perceived changes, assimilation, similarity and outcome. J Consult Clin Psychol. 1992;60:34-40.

24. Despland JN, de Roten Y, Despars J, Stigler M, Perry JC. Contribution of patient defense mechanisms and therapist interventions to the development of early therapeutic alliance in a brief psychodynamic investigation. J Psychoter Pract Res. 2001;10:155-64.

25. Ackerman SJ, Hilsenroth MJ. A review of therapist characteristics and techniques positively impacting the therapeutic alliance. Clin Psychol Rev. 2003;23:1-33.

26. Malan D. A study of brief psychotherapy. London: Tavistock; 1963.

27. Luborsky L, McLellan AT, Woody GE, O'Brien CP, Auerbach A. Therapist success and its determinants. Arch Gen Psychiatry. 1985;42:602-11.

28. Luborsky L, Mintz J, Auerbach A, Christoph P, Bachrach H, Todd $\mathrm{T}$, et al. Predicting the outcome of psychotherapy findings of the Penn Psychotherapy Project. Arch Gen Psychiatry. 1980;37:47181. 